OPEN ACCESS

Edited by:

Cynthia Calzas,

Institut National de la Recherche

Agronomique, France

Reviewed by:

Ralph A. Tripp,

University System of Georgia,

United States

Denis Archambault,

Université du Québec à

Montréal, Canada

*Correspondence:

Cyril Le Nouën

lenouenc@niaid.nih.gov

Specialty section:

This article was submitted to Vaccines and Molecular Therapeutics,

a section of the journal

Frontiers in Immunology

Received: 14 November 2018

Accepted: 16 May 2019

Published: 04 June 2019

Citation:

Le Nouën C, Collins PL and Buchholz UJ (2019) Attenuation of

Human Respiratory Viruses by

Synonymous Genome Recoding.

Front. Immunol. 10:1250

doi: 10.3389/fimmu.2019.01250

\section{Attenuation of Human Respiratory Viruses by Synonymous Genome Recoding}

\author{
Cyril Le Nouën*, Peter L. Collins and Ursula J. Buchholz \\ RNA Viruses Section, LID, NIAID, NIH, Bethesda, MD, United States
}

Using computer algorithms and commercial DNA synthesis, one or more ORFs of a microbial pathogen such as a virus can be recoded and deoptimized by several strategies that may involve the introduction of up to thousands of nucleotide (nt) changes without affecting amino acid (aa) coding. The synonymous recoding strategies that have been applied to RNA viruses include: deoptimization of codon or codon-pair usage, which may reduce protein expression among other effects; increased content of immunomodulatory $\mathrm{CpG}$ and UpA RNA, which increase immune responses and thereby restrict viral replication; and substitution of serine and leucine codons with synonymous codons for which single-nt substitutions can yield nonsense codons, thus limiting evolutionary potential. This can reduce pathogen fitness and create potential live-attenuated vaccines that may have improved properties. The combined approach of genome recoding, synthetic biology, and reverse genetics offers several advantages for the generation of attenuated RNA viruses. First, synonymous recoding involves many mutations, which should reduce the rate and magnitude of de-attenuation. Second, increasing the amount of recoding can provide increased attenuation. Third, because there are no changes at the aa level, all of the relevant epitopes should be expressed. Fourth, attenuation frequently does not compromise immunogenicity, suggesting that the recoded viruses have increased immunogenicity per infectious particle. Synonymous deoptimization approaches have been applied to two important human viral pathogens, namely respiratory syncytial virus (RSV) and influenza A virus (IAV). This manuscript will briefly review the use of these different methods of synonymous recoding to generate attenuated RSV and IAV strains. It also will review the characterization of these vaccine candidates in vitro and in animal models, and describe several surprising findings with respect to phenotypic and genetic instability of some of these candidates.

Keywords: human respiratory virus, respiratory syncytial virus, influenza virus, vaccine, genome recoding, synonymous codon deoptimization, synthetic biology

\section{INTRODUCTION}

The availability and affordability of large-scale commercial DNA synthesis opened the field of synthetic biology $(1,2)$. This technological advance allowed, in 2002, the rescue of an infectious poliovirus entirely from synthetic DNA (3). During the following years, synthetic biology and reverse genetics were combined to design and rescue viruses with extensive targeted modifications. 
This resulted, in 2006, in the rescue of poliovirus strains with extensive codon deoptimization (CD) $(4,5)$. This exemplifies the approach of synonymous genome recoding, in which one or more ORFs of a microbial pathogen are modified at the nt level without altering coding at the aa level. Subsequently, synonymous genome recoding has been widely applied to reduce pathogen fitness and create potential live-attenuated vaccines.

Deoptimization by synonymous genome recoding offers several advantages for viral vaccine design. Genomes of recoded viruses may contain up to thousands of synonymous nucleotide mutations in one or several ORFs. Many of these likely contribute to attenuation, in aggregate this large number should impose a significant barrier against reversion to virulence, because any single-site reversion likely would yield only a small amount of de-attenuation (6-8). In principle, the level of attenuation can be modulated by adjusting the number of introduced mutations. Recoded vaccine candidates encode all viral proteins with the same aa sequence as the wt parent, and thus should induce innate, humoral, and cell-mediated responses against the same array of epitopes. Recoded viruses also can contain an increased number of $\mathrm{CpG}$ and $\mathrm{UpA}$ RNA dinucleotides that may increase host immune responses that restrict the virus and provide greater efficacy. Because synonymous recoding does not involve the lengthy development of specific attenuating mutations, it provides an expedited means of developing attenuated strains of a known or newly emerging pathogen.

Synonymous genome recoding has been applied to two important human respiratory viruses with negative-sense RNA genomes, namely respiratory syncytial virus (RSV) and influenza virus type A (IAV). RSV belongs to the Pneumoviridae family and is the most important viral agent of severe respiratory illness in infants and young children worldwide, and also is an important cause of respiratory illness in the frail elderly. Vaccines or antiviral drugs suitable for routine use are not yet available. A live-attenuated vaccine is a strategy of choice for the pediatric population because it is free of RSV disease enhancement that is associated with inactivated and subunit RSV vaccines in RSV-naïve recipients. The RSV genome consists of a single nonsegmented negative-sense $15.2 \mathrm{~kb}$ RNA, containing 10 genes in the order $3^{\prime}$-NS1-NS2-N-P-M-SH-G-F-M2-L-5'. The M2 mRNA encodes two separate proteins, M2-1 and M2-2, from overlapping ORFs.

IAV belongs to the Orthomyxoviridae family, and contains eight RNA genome segments, each encoding one or two proteins: segment 1, PB2; 2, PB1 and PB1-F2; 3, PA, and PA$\mathrm{X}$ protein; 4, HA; 5, NP; 6, NA; 7, M1, and M2; 8, NS1. Antigenic change of IAV is driven by point mutations in the HA and NA proteins as well as segment reassortment. Three types of vaccines are currently licensed for IAV: inactivated, live-attenuated, and recombinant HA protein. This review describes the current strategies of synonymous genome recoding used to generate attenuated RSV and IAV viruses and the characterization in vitro and in vivo of the resulting vaccine candidates.

\section{FOUR STRATEGIES FOR SYNONYMOUS GENOME RECODING}

The four approaches used to deoptimize the different strains of IAV and RSV and the resulting number of silent nt mutations that have been introduced in these viruses ORFs are summarized in Table 1.

\section{Codon Deoptimization (CD)}

Due to the degeneracy of the genetic code, most amino acids are encoded by more than one nucleotide triplet (synonymous codons). Some codons are used more or less frequently than one would expect based on random chance. This unequal frequency of usage of synonymous codons, referred to as codon bias [CB, (19)], can be found in many organisms including viruses $(20,21)$. CD involves recoding part or all of one or more ORFs to increase the content of synonymous codons that normally are under-represented in the genome of these organisms.

Several hypotheses that have been proposed to explain $\mathrm{CB}$-as well as the effects of $\mathrm{CD}$-involve mechanisms by which $\mathrm{CB}$ might affect protein expression (usually to increase expression), and indeed there is a significant association between $\mathrm{CB}$ and translation efficiency in Escherichia. coli (E. coli) and Saccharomyces cerevisiae (22). One hypothesis is that the codon usage of a virus is adapted to the host tRNA abundance, thereby enhancing viral translation and fitness (21, 23-25). Indeed, in several prokaryotes and unicellular eukaryotes there is a consistent correlation between tRNA abundance and the corresponding codon usage frequency (26). This correlation is more difficult to establish for multicellular eukaryotes. In humans, the tRNA abundance varies widely among different tissues. However, the tRNA abundance was statistically correlated to codon usage of highly expressed genes specific for those tissues $(27,28)$. Nt assignments involved in mRNA secondary structures, and an avoidance of GC content, also might sometimes contribute to $\mathrm{CB}(19,29,30)$. Of note, the codon bias of negative strand RNA viruses frequently differs from that of their host. A recent study suggests that this discrepancy is due to constraints of the viral replication machinery; in a VSV minigenome system, the purine/pyrimidine content of viral RNAs affected the stability of the viral nucleocapsids and the RNA synthesis activity of viral polymerase complex (31). CB also has been suggested to occur as a means of regulating protein folding: for example, underrepresented tRNAs can decrease the rate of polypeptide chain elongation and thereby improve the quality of protein folding (32). In addition, selective pressure to reduce the content of CpG and UpA RNA, which appear to stimulate innate and adaptive immune responses that would restrict the virus, may contribute to CB (14).

\section{Codon-Pair Deoptimization (CPD)}

Just as codons may appear more or less frequently than expected, the usage of particular pairs of codons may be more or less frequent than expected which is called "codon pair bias" (CPB) (33). For example, in E. coli, two codon pairs encoding Leu-Ala [CTG-GCA and CTG-GCG] are highly overrepresented, while the synonymous codon pair CTG-GCC is under-represented. 
TABLE 1 | Attenuation of influenza and respiratory syncytial virus by synonymous genome recoding.

\begin{tabular}{|c|c|c|c|c|c|c|}
\hline Virus & $\begin{array}{l}\text { Deoptimization } \\
\text { strategy }\end{array}$ & Virus strain & Gene(s) deoptimized & $\begin{array}{l}\text { Number of silent } \\
\text { mutations }\end{array}$ & Main results & References \\
\hline \multirow[t]{4}{*}{ IAV } & $C D^{a}$ & Seasonal $\mathrm{H} 1 \mathrm{~N} 1$ & PB2, PB1, PA, HA, NP, NA, M, NS ${ }^{b}$ & $\begin{array}{l}62,77,65,46,31,47,27 \\
18\end{array}$ & $\begin{array}{l}\text { No effect on protein } \\
\text { expression in vitro } \\
\text { Virus attenuated in } \\
\text { mammalian cells and in } \\
\text { mice } \\
\text { Immunogenicity in mice } \\
\text { equivalent to wt }\end{array}$ & (9) \\
\hline & & PR8 H1N1 & NS & 135 & $\begin{array}{l}\text { Reduced NS1 and NEP } \\
\text { protein expression in vitro } \\
\text { Reduced virus replication in } \\
\text { vitro } \\
\text { Virus attenuated in mice } \\
\text { Immunogenicity in mice } \\
\text { equivalent to wt }\end{array}$ & (10) \\
\hline & & $2009 \mathrm{pH} 1 \mathrm{~N} 1^{\mathrm{d}}$ & $\mathrm{HA}, \mathrm{NA} \mathrm{e}^{\mathrm{e}}$ & 346,293 & $\begin{array}{l}\text { Reduced rate of replication } \\
\text { in vitro } \\
\text { Final titers in the lung of } \\
\text { ferrets equivalent to wt }\end{array}$ & (13) \\
\hline & $\begin{array}{l}\text { Increasing CpG or } \\
\text { UpA content }\end{array}$ & PR8 H1N1 & NP & $\begin{array}{l}86 \text { (CpG-high virus), } 73 \\
\text { (UpA-high virus) }\end{array}$ & $\begin{array}{l}\text { Reduced virus replication in } \\
\text { vitro } \\
\text { Virus attenuated in mice } \\
\text { Immunogenicity equivalent } \\
\text { to wt }\end{array}$ & (14) \\
\hline RSV & CPD & A2 & NS1, NS2, N, P, M, SH, G, F, Li & $\begin{array}{l}65,60,241,143,163,23 \\
197,422,1,378\end{array}$ & $\begin{array}{l}\text { Reduced protein expression } \\
\text { of CPD genes } \\
\text { Reduced virus replication in } \\
\text { vitro } \\
\text { Viruses attenuated in mice, } \\
\text { hamsters, and African green } \\
\text { monkeys } \\
\text { Immunogenicity equivalent } \\
\text { to wt }\end{array}$ & (18) \\
\hline
\end{tabular}

${ }^{a} C D$, codon deoptimization; $C P D$, codon-pair deoptimization.

${ }^{b}$ Recoded individually or in the combination of eight.

${ }^{c}$ Recoded individually and in combinations, notably NP-HA-PB1 (11) and HA-NA (12).

d2009 pandemic (p)H1N1.

${ }^{e}$ Recoded in combination.

${ }^{f}$ Serine and leucine codons recoded into synonymous codons for which some single-nt substitutions result in nonsense codons.

${ }^{\text {gRecoded separately. }}$

${ }^{h}$ Recoded in the combinations NS1-NS2 and NS1-NS2-G.

${ }^{i}$ Recoded in the combinations NS1-NS2-N-P-M-SH; G-F; L; and all genes except M2-1 and M2-2.

'Number of silent mutations introduced in each gene, respectively. 
While this non-randomness was evident for pairs of codons, a bias was much less obvious in control analyses evaluating pairs of non-adjacent codons, and almost absent when pairs were separated by two or three intervening codons (33). $\mathrm{CPB}$ is thought to affect translation due to differences in the compatibility of different synonymous pairs of aminoacyl-tRNAs in the translating ribosome $(33,34)$. Buchan and al. suggested that structural features that regulate tRNA geometry within the ribosome may favor specific codon pairs and thus govern genomic codon pair patterns, driving enhanced translational fidelity and/or rate (35). As with $\mathrm{CB}$, other factors may contribute to $\mathrm{CPB}$, such as selection to reduce the content of $\mathrm{CpG}$ and $\mathrm{UpA}$ RNA thought to be immunostimulatory (36). CPD is achieved by rearranging synonymous codons to increase the frequency of codon-pairs that typically are under-represented, without changing the overall codon usage or nt content. The first CPD of an RNA virus involved poliovirus, in 2008 (37).

\section{Increasing the $\mathrm{CpG}$ and UpA Content}

CpG and UpA RNAs typically are under-represented in RNA virus genomes, presumably due to selective pressure to reduce immune recognition by innate immunity sensors. CPD or CD of a viral genome frequently result in inadvertent increases in the $\mathrm{CpG}$ and UpA content of the recoded virus, which may account for the increased immunogenicity per PFU that sometimes is observed (11). For several RNA virus genomes, the content of CpG and UpA was deliberately increased while preserving the natural overall $\mathrm{CPB}$ and $\mathrm{CB}$ ratios (14,38-40). The resulting

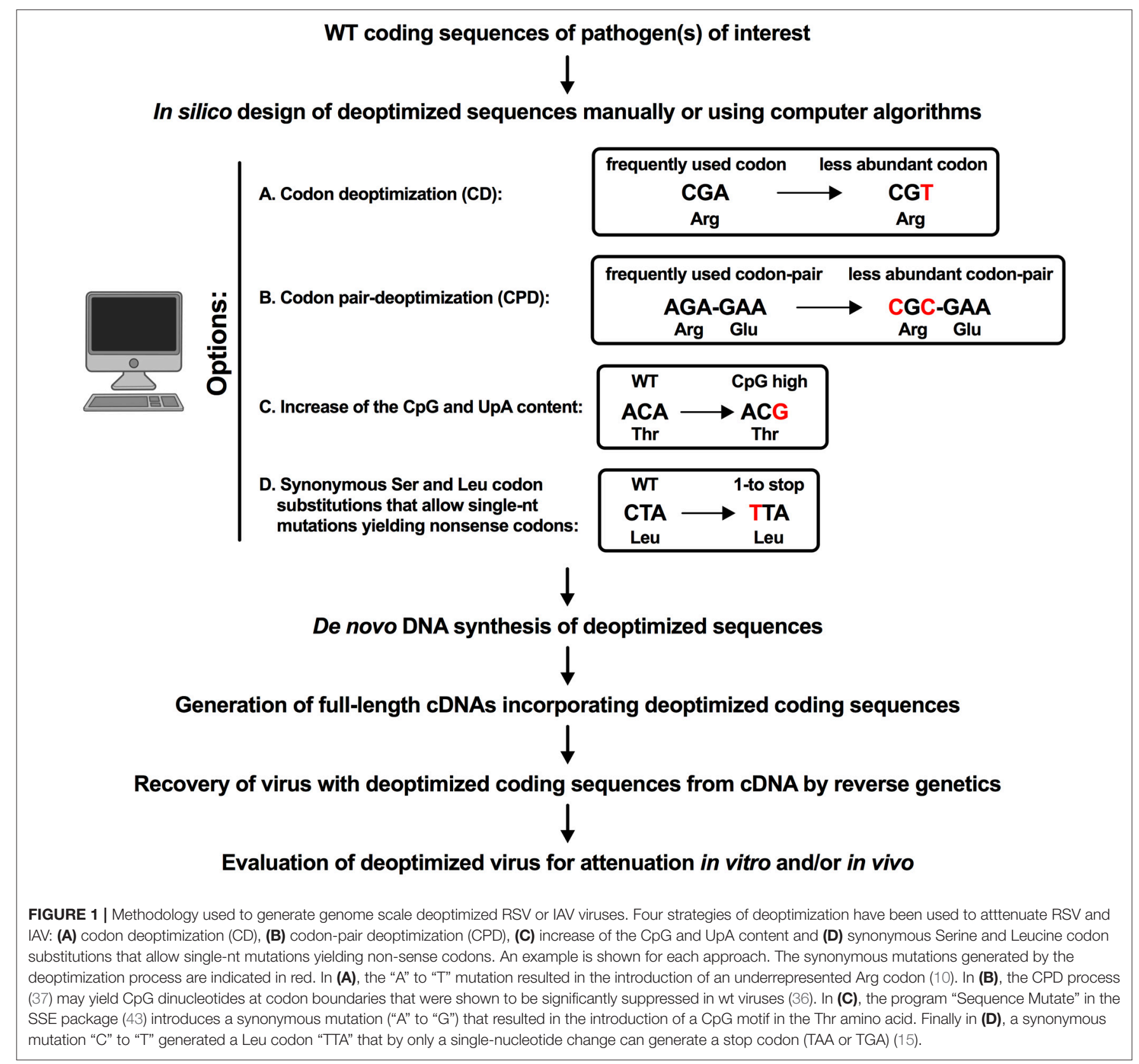


viruses were substantially attenuated yet were as immunogenic as their wt parents, presumably due to greater stimulation of innate immune sensors by $\mathrm{CpG}$ and $\mathrm{UpA}$ resulting in increased immune responses and restriction of virus replication.

Note that CpG DNA can directly and very efficiently activate B cells but also Natural Killer cells, dendritic cells and monocytes/macrophages through TLR9 stimulation (41). While this is less clear for CpG RNA, synthetic CpG RNAs have been shown to stimulate human monocytes resulting in IL-6 and IL-12 production and costimulatory molecule up-regulation. However, this effect is not mediated through TLR3, 7/8, or 9 and the pathways remain to be defined (42). In addition, during virus replication, large amounts of viral mRNAs or doublestranded intermediates are produced that could be potentially be recognized by sensors of the innate immune response. Whether or not the increased $\mathrm{CpG}$ and $\mathrm{UpA}$ content in recoded viruses results in increased immunogenicity remains to be determined.

Recently, the host Zinc-finger antiviral protein (ZAP) was shown to inhibit a recoded $\mathrm{CpG}$-rich version of $\mathrm{HIV}-1$ by directly binding to regions of the viral RNA rich in $\mathrm{CpG}$ RNA. This suggests that ZAP is part of the cellular system for detecting non-self RNA containing CpG RNA (40).

\section{Synonymous Serine and Leucine Codon Substitutions That Allow Single-nt Mutations Yielding Nonsense Codons}

Serine and leucine codons, which have the highest codon redundancy, can be recoded to increase the use of synonymous codons for which some of the possible single-nt changes result in nonsense mutations ("1-to-Stop" mutations) (15). This reduces the number of mutations at a given serine or leucine codon that can yield fit virus, and thus reduces evolutionary potential and viral fitness. This strategy has been applied to the pathogenic enterovirus Coxsackie B3 as well as to IAV (15).

\section{METHODOLOGY USED TO GENERATE DEOPTIMIZED RSV AND IAV VACCINE CANDIDATES}

The methodologies that have been applied to RSV or IAV to generate deoptimized vaccine candidates are described in Figure 1. In all approaches, following identification of the gene(s) or portion(s) of the ORF(s) that will be targeted for deoptimization, silent mutations were first introduced in silico, manually $(9,10,15)$ or using computer algorithms (11-14, 18), as described below. For each approach, packaging and splicing signals or replication/translation elements were excluded from the deoptimization process.

CD (Figure 1A) of the PR8 NS gene by Nogales and colleagues was done by introducing synonymous mutations to increase the abundance of codons that were under-represented in the natural coding sequences (10). Fan and colleagues used a different approach, as they changed the $\mathrm{CB}$ of a seasonal human $\mathrm{H} 1 \mathrm{~N} 1$ virus to an avian-like IAV CB (9). To do so, the authors first determined the segment specific CBs of the human H1N1 strain and the corresponding avian IAV sequences. This allowed to determine the number of mutations that had to be introduced into the human $\mathrm{H} 1 \mathrm{~N} 1 \mathrm{ORF}$ to change its $\mathrm{CB}$ to an avian IAV-like $\mathrm{CB}$. Mutations were randomly distributed in the targeted ORF at sites that were highly conserved at the amino acid sequence level to reduce the possibility of introducing mutations into potential mutational hot spots, or disrupting potential critical RNA signals. The free energy of the resulting RNAs and the dinucleotide usage frequency were unchanged by these modifications.

CPD (Figure 1B) was done using a computer algorithm to enrich a given viral coding sequence for codon-pairs underrepresented in a core set of human genes (37). First, the CPB of an ORF is defined by a codon pair score (CPS). This CPS is defined as the natural log of the ratio of the observed over the expected number of occurrences of each codon pair. The expected number of each codon pair was calculated so that it is independent both of the amino acid frequency and the codon bias (33). The CPB for an ORF was then determined as the arithmetic mean of all CPS. The CPD algorithm of Coleman and colleagues (37) shuffles the existing codons of an ORF to generate under represented codon pairs, while preserving the codon bias and the free energy of the folding of the recoded RNA.

The increase of the CpG and UpA content of IAV NP gene (Figure 1C) (14) was completed using the computer algorithm "Sequence Mutate" in the SSE package (43) while maintaining the mononucleotide composition through the introduction of compensatory substitutions elsewhere in the sequence. The $\mathrm{CPB}$ was not modified by this process.

Finally, Moratorio and colleagues introduced synonymous mutations that allow single-nt mutations yielding nonsense codons into the HA or PA gene of the 2009 pandemic H1N1 virus only in codons coding for two amino acids with the highest codon redundancy (Leu and Ser) to limit the overall change in nucleotide sequence to $<5 \%$. These Leu and Ser mutations did not affect the $\mathrm{CB}, \mathrm{CPB}, \mathrm{CpG}$ content or dinucleotide frequency (Figure 1D) (15).

In every case, the deoptimized sequences were synthetized de novo and cloned into plasmids that were used to rescue the deoptimized virus of interest by reverse genetics. The rescued viruses were then evaluated phenotypically in vitro and in vivo.

\section{SYNONYMOUS GENOME RECODING OF IAV}

\section{Codon Deoptimization (CD)}

$\mathrm{CD}$ was applied to the seasonal human H1N1 by converting its codon usage so that it was similar to that observed in avian influenza virus, in order to attenuate the virus for humans without reducing yield in embryonated chicken eggs, the substrate for vaccine production (9). All eight segments were codon-deoptimized alone or in combination. This attenuated the virus in mammalian cells and in mice, whereas replication in embryonated eggs remained comparable to wt. Surprisingly, CD did not affect protein expression (9), illustrating that its effects can be different than predicted.

CD also was applied to the laboratory-passaged H1N1 PR8 strain, involving only the NS gene (10). CD did not affect NS 
mRNA transcription in MDCK cells but, as expected, did reduce NS protein expression in MDCK and human airway A549 cells, and virus replication was reduced in A549 cells. The CD PR8 virus was attenuated in mice. Both the $\mathrm{CD}$ seasonal human H1N1 and the CD PR8 IAV retained their immunogenicity despite attenuation and conferred homologous and heterologous protection against IAV challenge in mice $(9,10)$.

\section{Codon-Pair Deoptimization (CPD)}

The NP, HA, NA, and PB1 segments of the laboratory-passaged PR8 H1N1 strain were subjected to CPD alone or in several combinations $(11,12)$. PR8 that contained CPD NP, HA, NA, and $\mathrm{PB} 1$ alone or in various combinations replicated to about 10 -fold lower titers than wt PR8 on MDCK cells. However, the replication of the PR8/CPD-HA-NA virus in human A549 cells was 1,000fold lower than wt. As expected, translation of the CPD genes was reduced compared to other genes from the same virus (11). Surprisingly, in case of the CPD NA mRNA, transcription was also reduced, with the underlying mechanism being unclear (12).

Despite overall robust replication in vitro, PR8 viruses with various combinations of CPD NP, HA, PB1 genes were attenuated in mice, and the attenuation increased with increasing number of CPD genome segments (11). In mice, the PR8/CPD-NP-HA$\mathrm{PB} 1$ virus did not induce any disease symptoms or weight loss and was 3,000-fold reduced for replication compared to wt. In addition, it was a more potent inducer of IAV-specific antibodies than wt, and replication of challenge virus was below the level of detection in $80 \%$ of the mice (11). The virus PR8/CPD-HANA also was attenuated in vivo, replicating to 100 - to 1,000 -fold lower titers than wt in the lungs of mice, with the NA gene being the major contributor of attenuation (12). This virus also induced a strong antibody response that was equivalent to wt, and it efficiently protected against lethal challenge, with protection being durable for at least 7 months (12).

Since CPD of the HA and NA genes was so highly attenuating for the PR8 virus, the HA, and NA genes of the 2009 pandemic (p)H1N1 strain similarly were subjected to CPD in combination (13). The resulting virus had a reduced rate of replication in MDCK cells, but final titers were similar to those of its pH1N1 parent (13). In ferrets, the CPD-HA-NA virus was nonpathogenic. However, no significant difference in virus titers in the lung at day 3 pi was observed between this virus and its pH1N1 parent, suggesting that additional genes will need to be subjected to CPD to obtain additional attenuation (13). Thus, there can be strain-to-strain variability in the attenuation achieved by CPD.

\section{Increasing the $\mathrm{CpG}$ and UpA Content}

Segment 5 (encoding NP) of IAV PR8 was recoded to increase the content of CpG or UpA RNA (14). Replication of the two resulting viruses (CpG-high or UpA-high) was delayed compared to wt in MDCK cells, with final titers that were about 10fold reduced. Plaque size and infectivity were also reduced. The viruses replicated to 10 -fold lower titers than their wt parent in mice, but cytokine production, CD4+, CD8+-T cell and antibody responses were comparable to those induced by wt virus, and the mutants were fully protective against wt challenge.
This suggested that CpG- and UpA-high IAV viruses may induce innate and adaptive immune responses disproportionate to their replication phenotypes (14).

\section{Synonymous Serine and Leucine Codon Substitutions That Allow Single-nt Mutations Yielding Nonsense Codons}

Moratorio and colleagues recoded either the HA or PA gene of the 2009 pandemic H1N1 virus to replace serine and leucine codons with synonymous codons for which a number of singlent substitutions could yield nonsense mutations (15). There was no effect of the recoding on virus replication in MDCK cells. However, both viruses exhibited an increase in nonsense mutations in the mutated genes compared to wt, that significantly reduced viral fitness. In addition, virus replication was reduced 10 - to 100 -fold in mice. Despite reduced replication, the antibody response was comparable to wt and these viruses induced complete protection against wt virus. The apparent greater immunogenicity per PFU was suggested to be due to immune stimulation by truncated proteins and by adjuvant effects of defective viruses (15). Thus, reducing the evolutionary potential of a virus provides a novel attenuation strategy.

\section{SYNONYMOUS GENOME RECODING OF RSV}

\section{Codon-Deoptimization (CD)}

$\mathrm{CD}$ was performed for the ORFs encoding the RSV interferon antagonist non-structural proteins 1 (NS1) and 2 (NS2) (16, $17,44,45)$ and the gene encoding the attachment glycoprotein G (17). As a result of CD, the level of NS1 and NS2 protein was reduced in Vero, BEAS-2B, and Hep-2 cells $(16,44)$ and the expression of $G$ was reduced in Vero cells (17). CD of NS1 and NS2 did not affect virus replication in Hep-2 and Vero cells, but significantly reduced virus replication in the interferon competent bronchial airway BEAS-2B cells, as well as in differentiated normal human bronchial epithelium (NHBE) cells grown at the air-liquid interface (ALI) (16). The addition of the CD G further reduced virus replication on NHBE/ALI cells, probably due to the role of $G$ in attachment to primary cells (17). Interestingly, while infection of human 293 cells with wt RSV induced a 50\% reduction of STAT2 expression, RSV/CD-NS1-NS2 had no effect on STAT2 levels, indicating that this virus had lost the ability to inhibit this aspect of innate immunity. Compared to wt RSV-infected cells, NF-kB activation was reduced in RSV/CD-NS1-NS2- infected cells. The reduced activation of NF-kB by this virus may increase cell apoptosis thus contributing to the attenuated phenotype of this virus (16).

RSV/CD-NS1-NS2 and RSV/CD-NS1-NS2-G replicated to 10 -fold lower titers in the lungs of mice compared to wt, but induced significantly higher level of antibodies, and animals were protected against challenge (16). The RSV/CD-NS1-NS2-G virus also was strongly attenuated in the upper and lower respiratory tract of cotton rats, but still induced high levels of antibodies and the vaccinated animals were completely protected against wt challenge (17). 


\section{Codon-Pair Deoptimization (CPD)}

Our group studied the effect of genome-scale CPD of RSV $(18,46)$. Four CPD RSV strains were designed in which one (L), two ( $\mathrm{G}$ and F), six (NS1, NS2, N, P, M, and SH), and nine (all except M2-1 and M2-2) ORFs were subjected to CPD. All four CPD RSVs were temperature sensitive, which is a novel and unexplained effect, but as one possibility might indicate deficiencies in protein folding resulting from altered translation of CPD ORFs. The viruses grew less efficiently than wt in vitro and had reduced $\mathrm{mRNA}$ and protein synthesis. CPD of the surface glycoproteins $G$ and $F$ resulted in the strongest reduction in virus replication. The CPD RSVs exhibited a level of attenuation in mice and African Green monkeys comparable with that of two attenuated RSV strains presently in clinical trials (18).

The RSV bearing nine CPD ORFs was phenotypically and genetically stable when subjected to serial passage in vitro at progressively increasing temperature. Serial passage at increasing temperature of the RSV bearing the CPD L ORF (Min L) induced a partial loss of temperature-sensitivity and the acquisition of a broad array of mutations that were predominantly missense. Surprisingly, many of the mutations involved ORFs other than L, suggestive of changes affecting protein interactions to compensate for the reduced quantity of L protein. Unexpectedly, each of two compensatory missense mutations in the M2-1 protein had a major effect on restoring viral fitness, which differs from the expectation that individual mutations would have modest effects on viral fitness. The introduction of several of the compensatory mutations identified in the passaged viruses into Min $\mathrm{L}$ resulted in increased genetic stability, and the resulting virus was strongly attenuated in vivo but was comparable to wt RSV in immunogenicity and protective efficacy, yielding an improved vaccine candidate (46).

\section{BENEFITS, POTENTIAL LIMITS, AND FUTURE OF SYNONYMOUS GENOME RECODING}

Genome scale deoptimization of RNA viruses resulted in the generation of vaccine candidates that in most cases were attenuated in vitro, and always attenuated in animal models. While this approach has many advantages that have been described above, the underlying mechanism and resultant effects of the deoptimization still need to be further explored.

Firstly, the extent of deoptimization tolerated by viruses differs widely from virus to virus. For example, while extensive CPD of RSV (up to 9 out the of 11 ORFs CPD) still readily generates a replicating virus, this is not the case for poliovirus or HIV, where extensive CPD or CD did not yield replicating virus. This renders the effect of deoptimization on viral genes hard to predict and implies that, in each case, phenotypes have to be evaluated experimentally. In addition, $\mathrm{CD}, \mathrm{CPD}$, and the increase of the $\mathrm{CpG}$ and UpA content in some cases resulted in the decrease of the specific infectivity of the recoded viruses. This effect varied depending of the virus and the genes that had been deoptimized and also has to be carefully evaluated on a case-by-case basis.
Most of the approaches described above share common features. For example, the increase of the CpG content was the intended effect in one approach (14), and an also a side effect of CPD. Translation efficiency is expected to be affected both by $\mathrm{CD}$ and CPD. With the exception of poliovirus, different deoptimization approaches have not been directly compared side by side using the same genes or portion of genes of a pathogen. This renders the comparison of the efficiency of the different strategies difficult. For poliovirus, both CD and CPD of the same region of the capsid-encoding ORF generated attenuated viruses $(5,37)$. Interestingly, while both approaches reduced the specific infectivity of the deoptimized viruses, CD reduced the specific infectivity 10 -fold more than $\mathrm{CPD}$, suggesting that $\mathrm{CD}$ might have a greater effect on the specific infectivity than CPD, at least for poliovirus.

A direct comparison of deoptimized vaccine candidates to those generated by traditional approaches (e.g., biological viruses attenuated by serial passage, recombinant viruses attenuated by gene or codon deletions or non-synonymous attenuating mutations) is also mostly lacking. Pre-clinical evaluation of CPD RSV vaccine candidates showed that the level of restriction of CPD RSVs in African green monkeys was similar to that of two live-attenuated pediatric RSV vaccine candidates presently in clinical trials in infants and young children (18).

Importantly, despite strong restriction of replication in vivo, the deoptimized viruses generally induced a strong immune response in vaccinated animals, usually at the level observed with wt virus. As mentioned above, large amounts of viral mRNAs or double-stranded intermediates are produced during virus replication that could be potentially be recognized by the innate immune sensors. It is tempting to speculate that the increased $\mathrm{CpG}$ and $\mathrm{UpA}$ content in the recoded viruses results in viral mRNAs and/or double double-stranded intermediates with increased immunogenicity. However, this hypothesis would need to be verified. A comprehensive evaluation of the activation and/or proliferation of immune cells (dendritic cells, CD4+, and CD8+ T cells) following stimulation with these viruses would be helpful to elucidate the basis of this strong immunogenicity. In addition, a comprehensive evaluation of the immune response of non-human primates would be informative. Evaluation of the attenuation and immunogenicity of deoptimized vaccine candidates in phase I studies will provide answers on the usefulness of these approaches. Finally, it would also be of interest to complete the reverse experiment by recoding viruses using the most used codon pairs or by further reducing the CpG and UpA content to investigate the resulting effect on virus replication and immunogenicity in animal models.

Overall, the data available to date encourage the further evaluation of these vaccine candidates in clinical trials. However, a more comprehensive understanding is needed of the mechanisms of attenuation conferred by the different strategies of deoptimization. De-attenuation appears to be rare, suggesting that these viruses are genetically stable $(4,5,16,17,37,47-$ 51). However, an important limitation is that the de-optimized viruses generally have not been subjected to strong selective pressure that would favor the outgrowth of viruses with deattenuating mutations. In the study in which strong selective 
pressure was applied to CPD RSV, the virus containing nine CPD ORFs was stable. However, selective pressure on the virus that contained only the CPD L ORF (Min L) resulted in a number of unexpected findings, including de-attenuating mutations outside the CPD ORF that presumably compensated for the low expression of the CPD genes. Importantly, those mutations were used to make a more stable and more immunogenic vaccine candidate. Thus, further studies will be needed to understand escape mechanisms from the restrictions imposed by CPD.

\section{CONCLUSION}

While genome scale deoptimization of RNA viruses was initiated a decade ago, most of the vaccine candidates generated to date have been evaluated only in animal models.

\section{REFERENCES}

1. Abil Z, Xiong X, Zhao H. Synthetic biology for therapeutic applications. Mol Pharm. (2015) 12:322-31. doi: 10.1021/mp500392q

2. Burbelo PD, Ching KH, Han BL, Klimavicz CM, Iadarola MJ. Synthetic biology for translational research. Am J Transl Res. (2010) 2:381-9.

3. Cello J, Paul AV, Wimmer E. Chemical synthesis of poliovirus cDNA: generation of infectious virus in the absence of natural template. Science. (2002) 297:1016-8. doi: 10.1126/science.1072266

4. Burns CC, Shaw J, Campagnoli R, Jorba J, Vincent A, Quay J, et al. Modulation of poliovirus replicative fitness in HeLa cells by deoptimization of synonymous codon usage in the capsid region. J Virol. (2006) 80:3259-72. doi: 10.1128/JVI.80.7.3259-3272.2006

5. Mueller S, Papamichail D, Coleman JR, Skiena S, Wimmer E. Reduction of the rate of poliovirus protein synthesis through large-scale codon deoptimization causes attenuation of viral virulence by lowering specific infectivity. $J$ Virol. (2006) 80:9687-96. doi: 10.1128/JVI.00738-06

6. Lauring AS, Jones JO, Andino R. Rationalizing the development of live attenuated virus vaccines. Nat Biotechnol. (2010) 28:573-9. doi: 10.1038/nbt.1635

7. Hanley KA. The double-edged sword: How evolution can make or break a live-attenuated virus vaccine. Evolution (NY). (2011) 4:635-43. doi: 10.1007/s12052-011-0365-y

8. Bull JJ. Evolutionary reversion of live viral vaccines: can genetic engineering subdue it? Virus Evol. (2015) 1:vev005. doi: 10.1093/ve/vev005

9. Fan RL, Valkenburg SA, Wong CK, Li OT, Nicholls JM, Rabadan R, et al. Generation of live attenuated influenza virus by using codon usage bias. $J$ Virol. (2015) 89:10762-73. doi: 10.1128/JVI.01443-15

10. Nogales A, Baker SF, Ortiz-Riano E, Dewhurst S, Topham DJ, MartinezSobrido L. Influenza A virus attenuation by codon deoptimization of the NS gene for vaccine development. J Virol. (2014) 88:10525-40. doi: 10.1128/JVI.01565-14

11. Mueller S, Coleman JR, Papamichail D, Ward CB, Nimnual A, Futcher B, et al. Live attenuated influenza virus vaccines by computer-aided rational design. Nat Biotechnol. (2010) 28:723-6. doi: 10.1038/nbt.1636

12. Yang C, Skiena S, Futcher B, Mueller S, Wimmer E. Deliberate reduction of hemagglutinin and neuraminidase expression of influenza virus leads to an ultraprotective live vaccine in mice. Proc Natl Acad Sci USA. (2013) 110:9481-6. doi: 10.1073/pnas.1307473110

13. Broadbent AJ, Santos CP, Anafu A, Wimmer E, Mueller S, Subbarao K. Evaluation of the attenuation, immunogenicity, and efficacy of a live virus vaccine generated by codon-pair bias de-optimization of the 2009 pandemic H1N1 influenza virus, in ferrets. Vaccine. (2015) 34:563-70. doi: 10.1016/j.vaccine.2015.11.054

14. Gaunt E, Wise HM, Zhang H, Lee LN, Atkinson NJ, Nicol MQ, et al. Elevation of $\mathrm{CpG}$ frequencies in influenza A genome attenuates
These synonymous recoding strategies may prove useful for developing novel live-attenuated vaccines, such as for pediatric respiratory RSV vaccines as well as for emerging highly pathogenic viruses.

\section{AUTHOR CONTRIBUTIONS}

All authors listed have made a substantial, direct and intellectual contribution to the work, and approved it for publication.

\section{ACKNOWLEDGMENTS}

This research was supported by the Intramural Research Program of the National Institute of Allergy and Infectious Diseases (NIAID), National Institutes of Health (NIH). pathogenicity but enhances host response to infection. Elife. (2016) 5:e12735 doi: $10.7554 /$ eLife. 12735

15. Moratorio G, Henningsson R, Barbezange C, Carrau L, Borderia AV, Blanc H, et al. Attenuation of RNA viruses by redirecting their evolution in sequence space. Nat Microbiol. (2017) 2:17088. doi: 10.1038/nmicrobiol.2017.88

16. Meng J, Lee S, Hotard AL, Moore ML. Refining the balance of attenuation and immunogenicity of respiratory syncytial virus by targeted codon deoptimization of virulence genes. MBio. (2014) 5:e01704-14. doi: $10.1128 / \mathrm{mBio}$.01704-14

17. Stobart CC, Rostad CA, Ke Z, Dillard RS, Hampton CM, Strauss JD, et al. A live RSV vaccine with engineered thermostability is immunogenic in cotton rats despite high attenuation. Nat Commun. (2016) 7:13916. doi: $10.1038 /$ ncomms13916

18. Le Nouen C, Brock LG, Luongo C, McCarty T, Yang L, Mehedi M, et al. Attenuation of human respiratory syncytial virus by genome-scale codon-pair deoptimization. Proc Natl Acad Sci USA. (2014) 111:13169-74. doi: 10.1073/pnas.1411290111

19. Plotkin JB, Kudla G. Synonymous but not the same: the causes and consequences of codon bias. Nat Rev Genet. (2011) 12:32-42. doi: $10.1038 / \mathrm{nrg} 2899$

20. Goni N, Iriarte A, Comas V, Sonora M, Moreno P, Moratorio G, et al. Pandemic influenza A virus codon usage revisited: biases, adaptation and implications for vaccine strain development. Virol J. (2012) 9:263. doi: 10.1186/1743-422X-9-263

21. Wong EH, Smith DK, Rabadan R, Peiris M, Poon LL. Codon usage bias and the evolution of influenza A viruses. codon usage biases of influenza virus. BMC Evol Biol. (2010) 10:253. doi: 10.1186/ 1471-2148-10-253

22. Tuller T, Waldman YY, Kupiec M, Ruppin E. Translation efficiency is determined by both codon bias and folding energy. Proc Natl Acad Sci USA. (2010) 107:3645-50. doi: 10.1073/pnas.0909910107

23. Jenkins GM, Holmes EC. The extent of codon usage bias in human RNA viruses and its evolutionary origin. Virus Res. (2003) 92:1-7. doi: 10.1016/S0168-1702(02)00309-X

24. Hershberg R, Petrov DA. Selection on codon bias. Annu Rev Genet. (2008) 42:287-99. doi: 10.1146/annurev.genet.42.110807.091442

25. Aragones L, Guix S, Ribes E, Bosch A, Pinto RM. Fine-tuning translation kinetics selection as the driving force of codon usage bias in the hepatitis A virus capsid. PLoS Pathog. (2010) 6:e1000797. doi: 10.1371/journal.ppat.1000797

26. Ikemura T. Codon usage and tRNA content in unicellular and multicellular organisms. Mol Biol Evol. (1985) 2:13-34. doi: 10.1093/oxfordjournals.molbev.a040335

27. Dittmar KA, Goodenbour JM, Pan T. Tissue-specific differences in human transfer RNA expression. PLoS Genet. (2006) 2:e221. doi: 10.1371/journal.pgen.0020221 
28. Quax TE, Claassens NJ, Soll D, van der Oost J. Codon bias as a means to fine-tune gene expression. Mol Cell. (2015) 59:149-61. doi: 10.1016/j.molcel.2015.05.035

29. Kozak M. Pushing the limits of the scanning mechanism for initiation of translation. Gene. (2002) 299:1-34. doi: 10.1016/ S0378-1119(02)01056-9

30. Shabalina SA, Ogurtsov AY, Spiridonov NA. A periodic pattern of mRNA secondary structure created by the genetic code. Nucleic Acids Res. (2006) 34:2428-37. doi: 10.1093/nar/gkl287

31. Gumper RH, Li W, Luo M. Constraints of Viral RNA synthesis on codon usage of negative-strand RNA virus. J Virol. (2019) 93:e01775-18. doi: 10.1128/JVI.01775-18

32. Gustafsson C, Govindarajan S, Minshull J. Codon bias and heterologous protein expression. Trends Biotechnol. (2004) 22:346-53. doi: 10.1016/j.tibtech.2004.04.006

33. Gutman GA, Hatfield GW. Nonrandom utilization of codon pairs in Escherichia coli. Proc Natl Acad Sci USA. (1989) 86:3699-703. doi: 10.1073/pnas.86.10.3699

34. Gamble CE, Brule CE, Dean KM, Fields S, Grayhack EJ. Adjacent codons act in concert to modulate translation efficiency in yeast. Cell. (2016) 166:679-90. doi: 10.1016/j.cell.2016.05.070

35. Buchan JR, Aucott LS, Stansfield I. tRNA properties help shape codon pair preferences in open reading frames. Nucleic Acids Res. (2006) 34:1015-27. doi: 10.1093/nar/gkj488

36. Kunec D, Osterrieder N. Codon pair bias is a direct consequence of dinucleotide bias. Cell Rep. (2015) 14:55-67. doi: 10.1016/j.celrep.2015.12.011

37. Coleman JR, Papamichail D, Skiena S, Futcher B, Wimmer E, Mueller S. Virus attenuation by genome-scale changes in codon pair bias. Science. (2008) 320:1784-7. doi: 10.1126/science.1155761

38. Tulloch F, Atkinson NJ, Evans DJ, Ryan MD, Simmonds P. RNA virus attenuation by codon pair deoptimisation is an artefact of increases in CpG/UpA dinucleotide frequencies. Elife. (2014) 3:e04531. doi: 10.7554/eLife.04531

39. Atkinson NJ, Witteveldt J, Evans DJ, Simmonds P. The influence of CpG and UpA dinucleotide frequencies on RNA virus replication and characterization of the innate cellular pathways underlying virus attenuation and enhanced replication. Nucleic Acids Res. (2014) 42:4527-45. doi: 10.1093/nar/gku075

40. Takata MA, Goncalves-Carneiro D, Zang TM, Soll SJ, York A, Blanco-Melo D, Bieniasz PD. CG dinucleotide suppression enables antiviral defence targeting non-self RNA. Nature. (2017) 550:124-7. doi: 10.1038/nature24039

41. Krieg AM. CpG motifs in bacterial DNA and their immune effects. Annu Rev Immunol. (2002) 20:709-60. doi: 10.1146/annurev.immunol.20.100301.064842

42. Sugiyama T, Gursel M, Takeshita F, Coban C, Conover J, Kaisho T, et al. CpG RNA: identification of novel single-stranded RNA that stimulates human CD14+CD11c+ monocytes. J Immunol. (2005) 174:22739. doi: 10.4049/jimmunol.174.4.2273

43. Simmonds P. SSE: a nucleotide and amino acid sequence analysis platform. BMC Res Notes. (2012) 5:50. doi: 10.1186/1756-0500-5-50

44. Rostad CA, Stobart CC, Gilbert BE, Pickles RJ, Hotard AL, Meng J, et al. A recombinant respiratory syncytial virus vaccine candidate attenuated by a low-fusion $\mathrm{F}$ protein is immunogenic and protective against challenge in cotton rats. J Virol. (2016) 90:7508-18. doi: 10.1128/JVI.00012-16

45. Rostad CA, Stobart CC, Todd SO, Molina SA, Lee S, Blanco JCG, et al. Enhancing the thermostability and immunogenicity of a respiratory syncytial virus (RSV) live-attenuated vaccine by incorporating unique RSV line19f protein residues. J Virol. (2018) 92:e01568-17. doi: 10.1128/JVI.01568-17

46. Le Nouen C, McCarty T, Brown M, Smith ML, Lleras R, Dolan MA, et al. Genetic stability of genome-scale deoptimized RNA virus vaccine candidates under selective pressure. Proc Natl Acad Sci USA. (2017) 114:E386-95. doi: $10.1073 /$ pnas.1619242114

47. Bull JJ, Molineux IJ, Wilke CO. Slow fitness recovery in a codon-modified viral genome. Mol Biol Evol. (2012) 29:2997-3004. doi: 10.1093/molbev/mss119

48. Nougairede A, De Fabritus L, Aubry F, Gould EA, Holmes EC, de Lamballerie $\mathrm{X}$. Random codon re-encoding induces stable reduction of replicative fitness of Chikungunya virus in primate and mosquito cells. PLoS Pathog. (2013) 9:e1003172. doi: 10.1371/journal.ppat.1003172

49. Vabret N, Bailly-Bechet M, Lepelley A, Najburg V, Schwartz O, Verrier B, et all. Large-scale nucleotide optimization of simian immunodeficiency virus reduces its capacity to stimulate type I interferon in vitro. J Virol. (2014) 88:4161-72. doi: 10.1128/JVI.03223-13

50. Ni YY, Zhao Z, Opriessnig T, Subramaniam S, Zhou L, Cao D, et al. Computeraided codon-pairs deoptimization of the major envelope GP5 gene attenuates porcine reproductive and respiratory syndrome virus. Virology. (2014) 450451:132-9. doi: 10.1016/j.virol.2013.12.009

51. Cheng BY, Ortiz-Riano E, Nogales A, de la Torre JC, Martinez-Sobrido L. Development of live-attenuated arenavirus vaccines based on codon deoptimization. J Virol. (2015) 89:3523-33. doi: 10.1128/JVI.03401-14

Conflict of Interest Statement: CL, PC, and UB are coinventors on a patent application for the development of respiratory syncytial virus (RSV) vaccines by codon pair deoptimization.

Copyright (c) 2019 Le Nouën, Collins and Buchholz. This is an open-access article distributed under the terms of the Creative Commons Attribution License (CC BY). The use, distribution or reproduction in other forums is permitted, provided the original author(s) and the copyright owner(s) are credited and that the original publication in this journal is cited, in accordance with accepted academic practice. No use, distribution or reproduction is permitted which does not comply with these terms. 